\title{
Adsorption of Polyaromatic Backbone Impacts the Performance of Anion Exchange Membrane Fuel Cells
}

\author{
Ivana Matanovic, ${ }^{\$[\mathrm{a}, \mathrm{b}]}$ Sandip Maurya ${ }^{\sharp[\mathrm{c}]}$, Eun Joo Park ${ }^{[\mathrm{c}]}$, Jong Yeob Jeon ${ }^{[\mathrm{d}]}$, Chulsung Bae $^{[\mathrm{d}]}$, and Yu \\ Seung $\operatorname{Kim}^{*[\mathrm{c}]}$ \\ [a] Department of Chemical and Biological Engineering, Center for Micro-Engineered Materials (CMEM), University of \\ New Mexico, Albuquerque, New Mexico 87231 USA \\ [b] T-1: Physics and Chemistry of Materials, Los Alamos National Laboratory, Los Alamos, New Mexico 87545 USA \\ [c] MPA-11: Materials Synthesis and Integrated Devices, Los Alamos National Laboratory, Los Alamos, New Mexico \\ 87545 USA \\ [d] Department of Chemistry and Chemical Biology, Rensselaer Polytechnic Institute, Troy, New York 12180, USA
}

\section{Comparison in the performance of tested DFT functionals}

Table S1 shows that the absolute values for the adsorption energy of benzene on the Pt(111) surface differ among the used functionals ranging in value from $-1.00 \mathrm{eV}$ to $-1.91 \mathrm{eV}$. PW91 functional predicts the benzene adsorption energy of $-1.62 \mathrm{eV}$, which is in good agreement with the experimental value of $-1.57--1.86 \mathrm{eV}$ (for $0.5 \mathrm{ML}$ and $0.25 \mathrm{ML}$ coverage). ${ }^{1} \mathrm{The} v \mathrm{vW}$-DF of Dion, on the other hand, yields $-1.00 \mathrm{eV}$ for the benzene adsorption energy, a value that is lower than the PW91 adsorption energy, while the optPBE-vdW functional predicts benzene adsorption energy of $-1.95 \mathrm{eV}$. optPBE-vdW value is higher than the PW91 value but is in better agreement with the experimentally determined heat of adsorption of benzene on $\mathrm{Pt}(111)$ corresponding to the $0.25 \mathrm{ML}$ coverage. ${ }^{1}$ Please note that the adsorption of benzene is coverage dependent and the adsorption energy of benzene decreases as the benzene coverage increases, which is attributed to the repulsive lateral interactions between the adsorbed benzene molecules and a change in the adsorption sites as the coverage increases. ${ }^{1}$ Therefore, when comparing adsorption energies reported in different works, data have to be compared for similar coverage and same adsorption sites.

The comparison between the calculated and the experimental data for benzene adsorption on Pt(111) shows that the optimized $\mathrm{vdW}$ functionals, in which the original GGA functional was replaced with its optimized version, can effectively and accurately predict the interactions in which the bonding is largely contributed by the long-range correlation effects. We also point to a more extensive study concerning the ability of different vdW-inclusive DFT schemes to accurately describe the role of van der Waals forces in the benzene adsorption on various transition metal (111) surfaces. ${ }^{2}$ Comparing a larger number of transition metal surfaces and DFT functionals, this work also demonstrated poor performance of the vdW-DF functional and superior accuracy of the optimized exchange functionals (optPBE, optB88, and optB86b). The poor performance of the vdW-DF functional was attributed to the poor description of the short-range Pauli repulsion, which is found to be critical to accurately describe not only the weakly bound systems (physisorption) but also the strongly bound systems (chemisorption).

Adsorption energies of biphenyl calculated with different DFT functionals change in the same order as for the benzene adsorption. Namely, the adsorption energy of biphenyl calculated using different DFT functionals increases in the order of vdW-DF (-1.21 eV) $<$ PW91 $(-2.01 \mathrm{eV})<$ optPBE-vdW $(-2.87 \mathrm{eV})$.

Although the calculated absolute adsorption energies differ depending on the used functional (Table S2), the relative adsorption energies of three structural isomers of therphenyl are consistent among all the functionals. The same is true for fluorene and biphenyl ether. The exception is vdW-DF functional, which predicts $o$-terphenyl and 9,9-dimethyl fluorene to have adsorption energy similar to that of benzene, while both PW91 and optPBE-vdW predict $o$-terphenyl and 9,9-dimethyl fluorene have smaller adsorption energy than benzene. Also, notice that for $o$-terphenyl and 9,9-dimethyl fluorene PW91 functional predicts very low adsorption energies ($0.14 \mathrm{eV}$ and $-0.03 \mathrm{eV})$, while vdW-DF and optPBE-vdW predict higher adsorption energies $(>1 \mathrm{eV})$. 
Table S1. Adsorption energies (in eV) of different benzene-containing fragments on Pt(111) calculated using different DFT functionals.

\begin{tabular}{|c|c|c|c|}
\hline & PW91 & vdW-DF & optPBE-vdW \\
\hline benzene & -1.62 & -1.00 & -1.95 \\
biphenyl & -2.01 & -1.21 & -1.52 \\
$o$-terphenyl & -0.14 & -1.04 & -3.61 \\
$m$-terphenyl & -2.35 & -1.30 & -3.94 \\
$p$-terphenyl & -2.64 & -1.49 & -2.19 \\
diphenyl ether & -1.76 & -1.20 & -1.38 \\
9,9-dimethy fluorene & -0.03 & -1.06 & \\
\hline
\end{tabular}



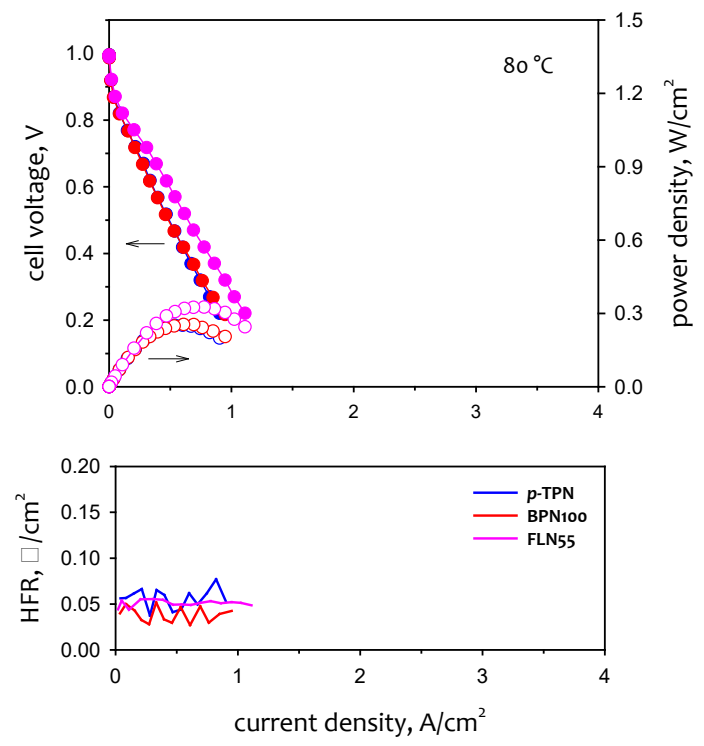

Figure S1. Effect of cathode ionomer on AEMFC performance at $80{ }^{\circ} \mathrm{C}$ with fully humidified $\mathrm{H}_{2}(2000 \mathrm{sccm})$ and $\mathrm{O}_{2}$ $(1000 \mathrm{sccm})$ at $285 \mathrm{kPa}$ backpressure. Anode: $\mathrm{Pt} / \mathrm{C}\left(0.6 \mathrm{mg}_{\mathrm{Pt}} \mathrm{cm}^{-2}\right)$, cathode: $\mathrm{Pt} / \mathrm{C}\left(0.6 \mathrm{mg}_{\mathrm{Pt}} \mathrm{cm}^{-2}\right)$, anode ionomer: $p$ TPN and cathode ionomer: $p$-TPN, BPN100 or FLN55, and $m$-TPN membrane (thickness: $30 \pm 5 \mu \mathrm{m}$ ). 

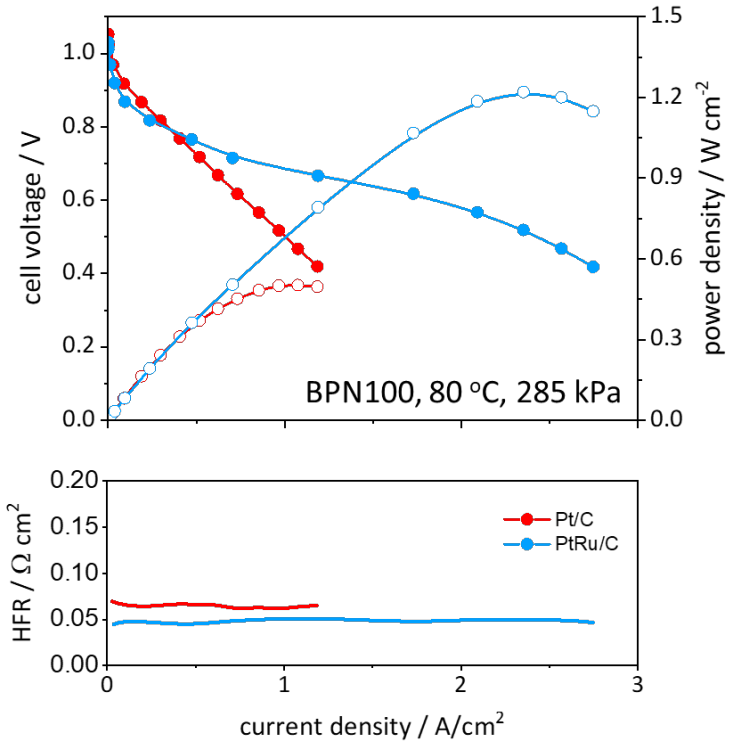

Figure S2. Impact of HOR catalysts on AEMFC performance. Anode: Pt/C (0.6 mg $\left.\mathrm{mg}_{\mathrm{Pt}} / \mathrm{cm}^{2}\right)$ or PtRu/C $\left(0.5 \mathrm{mgg}_{\mathrm{Pt}} / \mathrm{cm}^{2}\right)$, Cathode: $\mathrm{Pt} / \mathrm{C}\left(0.6 \mathrm{mg}_{\mathrm{Pt}} / \mathrm{cm}^{2}\right)$, and BPN100 ionomer. All fuel cell performances were obtained with fully humidified $2000 \mathrm{sccm} \mathrm{H}_{2}$ and $1000 \mathrm{sccm} \mathrm{O}_{2}$ at $285 \mathrm{kPa}$ backpressure and $m$-TPN membrane (thickness: $30 \pm 5 \mu \mathrm{m}$ ).

a)

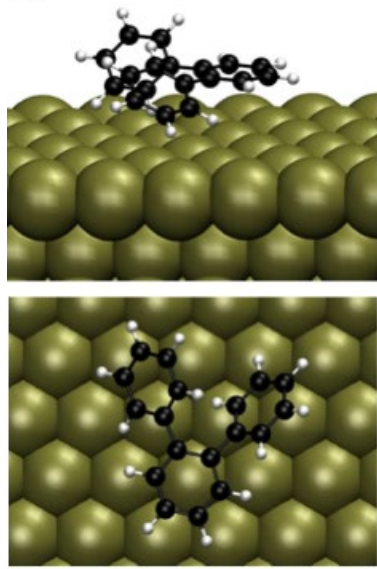

$\Delta E_{\mathrm{ad}}=-1.52 \mathrm{eV}$ b)
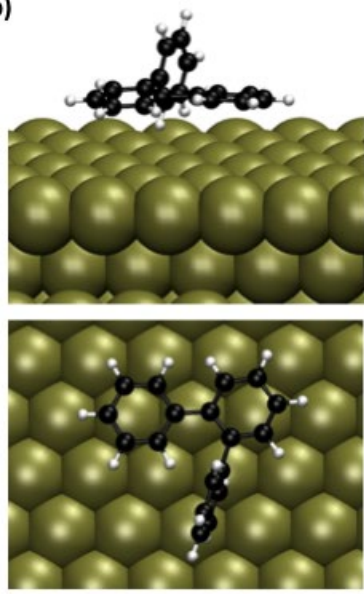

$\Delta E_{\mathrm{ad}}=-1.48 \mathrm{eV}$ c)
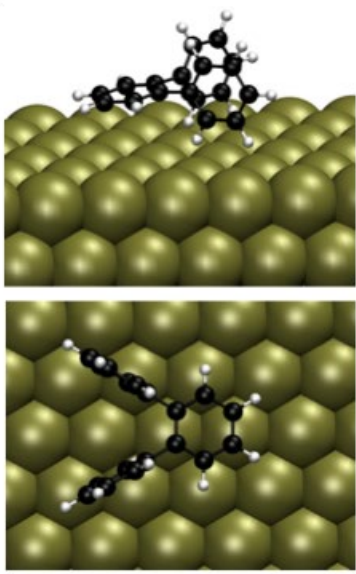

$\Delta E_{\mathrm{ad}}=-1.11 \mathrm{eV}$

Figure S3. DFT optimized geometries of different conformations of $o$-terphenyl adsorbed on the $\operatorname{Pt}(111)$ surface: a)

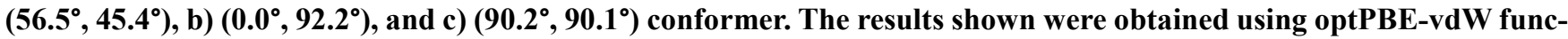
tional. 
a)
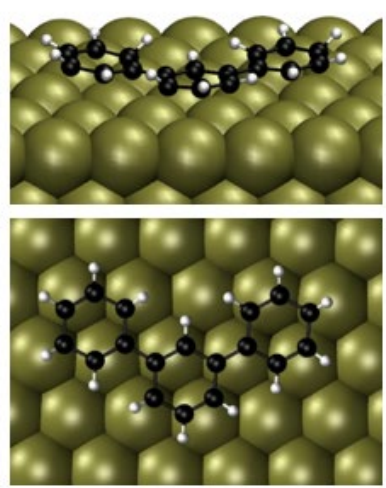

$\Delta E_{\mathrm{ad}}=-3.61 \mathrm{eV}$ b)
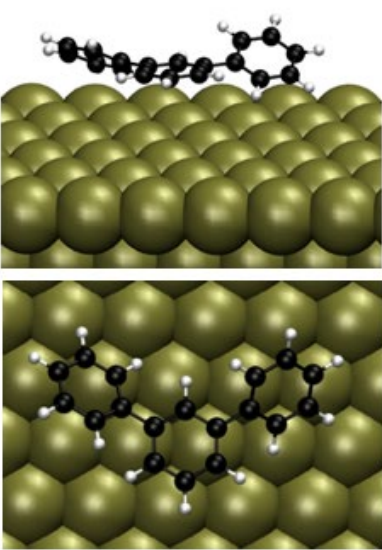

$\Delta E_{\mathrm{ad}}=-1.60 \mathrm{eV}$ c)
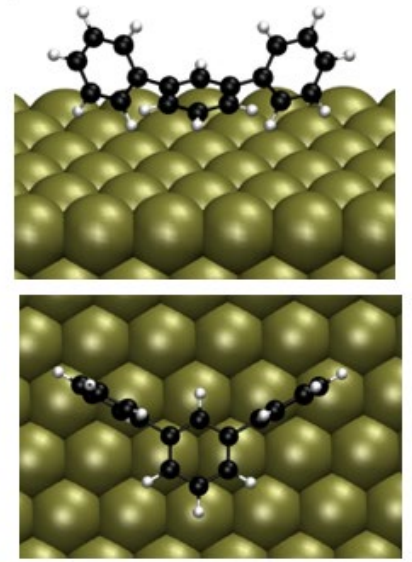

$\Delta E_{\mathrm{ad}}=-0.90 \mathrm{eV}$

Figure S4. DFT optimized geometries of different conformations of $\boldsymbol{m}$-terphenyl adsorbed on the Pt(111) surface: a) $\left(0.1^{\circ}, 0.0^{\circ}\right)$, b) $\left(43.2^{\circ}, 43.4^{\circ}\right)$, and c) $\left(89.7^{\circ}, 89.8^{\circ}\right)$ conformer. The results shown were obtained using optPBE-vdW functional.

a)
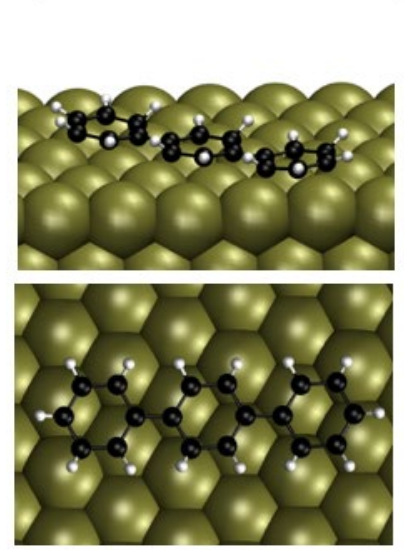

$\Delta E_{\mathrm{ad}}=-3.94 \mathrm{eV}$ b)
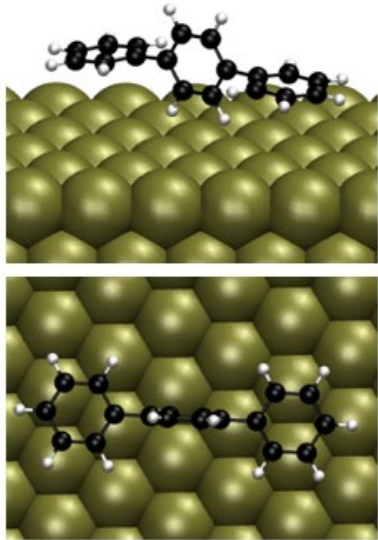

$\Delta E_{\mathrm{ad}}=-1.79 \mathrm{eV}$ c)
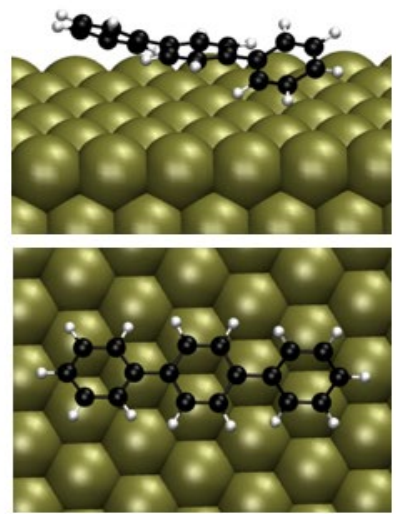

$\Delta E_{\mathrm{ad}}=-1.02 \mathrm{eV}$

Figure S5. DFT optimized geometries of different conformations of $p$-terphenyl adsorbed on the Pt(111) surface: a) $\left(0.1^{\circ}, 0.0^{\circ}\right)$, b) $\left(43.6^{\circ}, 43.8^{\circ}\right)$, and c) $\left(89.3^{\circ}, 89.9^{\circ}\right)$ conformer. The results shown were obtained using optPBE-vdW functional. 
a)
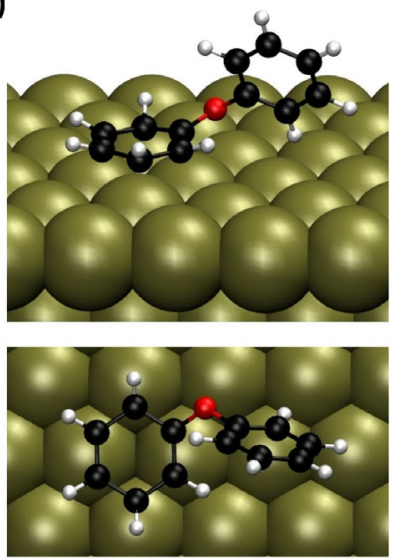

b)
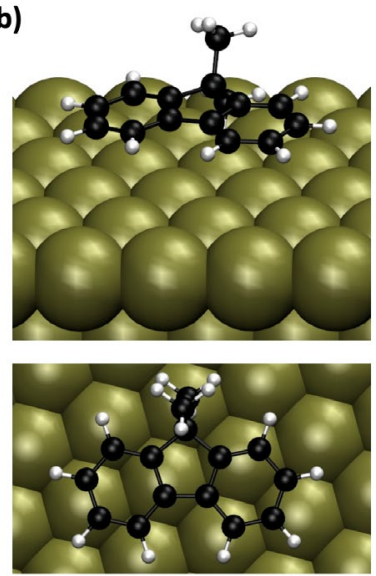

Figure S6. DFT optimized geometries of a) biphenyl ether and b) fluorene adsorbed on the Pt(111) surface as obtained using optPBE-vdW functional.
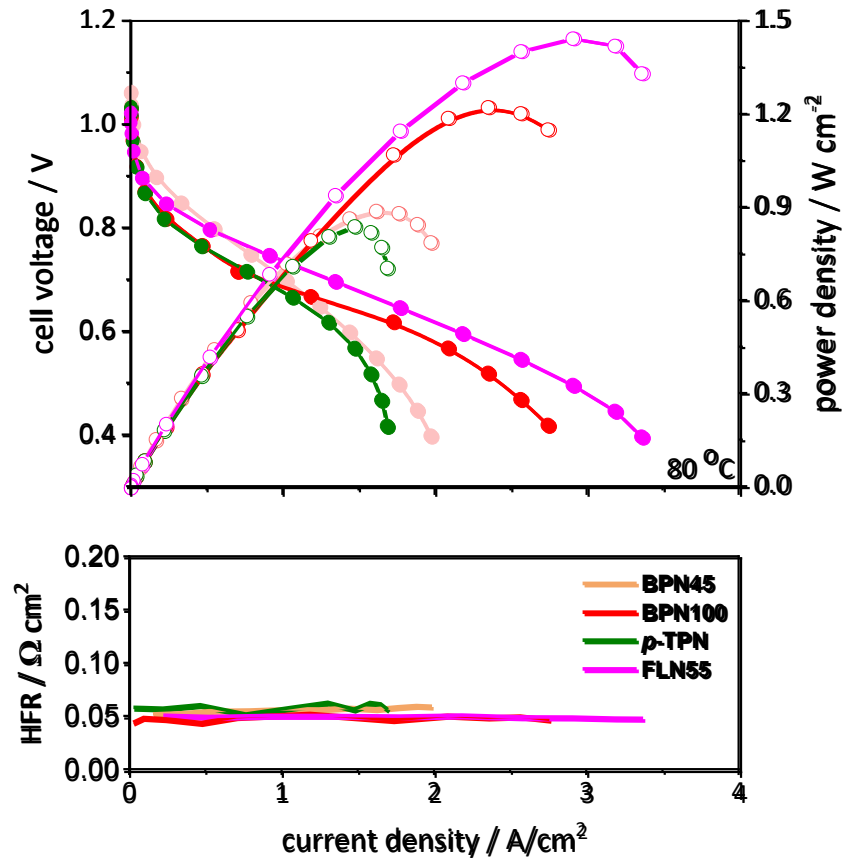

Figure S7. AEMFC performance with PtRu/C $\left(0.5 \mathrm{mgPt} / \mathrm{cm}^{2}\right)$ as an anode and $\mathrm{Pt} / \mathrm{C}\left(0.6 \mathrm{mgPt} / \mathrm{cm}^{2}\right)$ as a cathode using BPN100, BPN45, $p$-TPN and FLN55 ionomers. All fuel cells performances were obtained with fully humidified 2000 sccm $\mathrm{H}_{2}$ and $1000 \mathrm{sccm} \mathrm{O}_{2}$ at $285 \mathrm{kPa}$ backpressure and $m$-TPN membrane (thickness: $30 \pm 5 \mu \mathrm{m}$ ).

\section{References}

1. Ihm, H.; Ajo, H. M.; Gottfried, J. M.; Bera, P.; Campbell, C. T., Calorimetric Measurement of the Heat of Adsorption of Benzene on Pt(111). J. Phys. Chem. B 2004, 108 (38), 14627-14633.

2. Carrasco, J.; Liu, W.; Michaelides, A.; Tkatchenko, A., Insight into the Description of van der Waals Forces for Benzene Adsorption on Transition Metal (111) Surfaces. J. Chem. Phys. 2014, 140 (8), 084704 (1-10). 